\title{
Doubly heavy baryons at the LHC
}

\author{
A. V. Berezhnoy, ${ }^{1, *}$ A. K. Likhoded ${ }^{2,3, \dagger}$ and A. V. Luchinsky ${ }^{2, \$}$ \\ ${ }^{1}$ SINP of Moscow State University, Moscow 119991, Russia \\ ${ }^{2}$ Institute for High Energy Physics, Protvino 142281, Russia \\ ${ }^{3}$ Moscow Institute of Physics and Technology, Dolgoprudny 141701, Russia
}

(Received 8 October 2018; published 12 December 2018)

\begin{abstract}
The theoretical analysis of production, lifetime, and decays of doubly heavy baryons is presented. The lifetime of $\Xi_{c c}^{++}$baryons recently measured by the LHCb Collaboration is used to estimate the lifetimes of other doubly heavy baryons. The production and the possibility of observation of $\Xi_{b c}$ baryons at the LHC are discussed.
\end{abstract}

DOI: 10.1103/PhysRevD.98.113004

\section{INTRODUCTION}

Doubly heavy baryons are extremely interesting objects that allow us to take a fresh look at the problems of the production and hadronization of heavy quarks. These baryons consist of two heavy and one light quark and therefore, unlike ordinary heavy baryons, are characterized by several scales at once:

$$
m_{Q_{1,2}} \gg m_{Q_{1}} \cdot v, \quad m_{Q_{2}} \cdot v \gg \Lambda_{\mathrm{QCD}},
$$

where $m_{Q_{1}}, m_{Q_{2}}$ are masses of heavy quarks, and $v$ is the velocity inside the quarkonium. For clarity, one can go to the coordinate representation and select a specific family of baryons. Thus, for a baryon $\Xi_{b c}$ containing $b$ and $c$ quarks simultaneously, the scales are ordered as follows:

$$
\lambda_{b}: \lambda_{c}: r_{b c}: r_{\mathrm{QCD}} \approx 1: 3: 9: 27,
$$

where $\lambda_{Q}=1 / m_{Q}$ is a Compton length of a quark, $r_{b c} \sim$ $1 /\left(v \cdot m_{Q}\right)$ is the heavy quark size, and $r_{\mathrm{QCD}}=\Lambda_{\mathrm{QCD}}$ is a scale of nonperturbative confinement [1].

It is worth mentioning that a baryon with one heavy quark is characterized by only two scales, namely, the mass of the heavy quark and $\Lambda_{\mathrm{QCD}}$. In the limit $m_{Q_{1}}, m_{Q_{2}} \rightarrow \infty$ a heavy diquark interacts with a light quark as a heavy antiquark and, therefore, it is quite natural to subdivide calculating the characteristics of doubly heavy quarkonium

\footnotetext{
*Alexander.Berezhnoy@cern.ch

Anatolii.Likhoded@ihep.ru

¥alexey.luchinsky@ihep.ru
}

Published by the American Physical Society under the terms of the Creative Commons Attribution 4.0 International license. Further distribution of this work must maintain attribution to the author(s) and the published article's title, journal citation, and DOI. Funded by SCOAP. in two stages: the calculation of the properties of the heavy diquark and the subsequent calculation of the properties of the quark-diquark system. ${ }^{1}$

The problems of production and decays of such systems have been of interest to researchers for many years. But the last year was special because it was marked by the discovery of the doubly charmed $\Xi_{c c}^{++}$baryon in the decay mode $\Lambda_{c}^{+} K^{-} \pi^{+} \pi^{+}$[4]. The LHCb Collaboration has observed hundreds of such particles. This discovery was confirmed by the observation of decay $\Xi_{c c}^{++} \rightarrow \Xi_{c}^{+} \pi^{+}$[5]. This circumstance greatly revived the research activities in this direction. In this article we discuss the perspectives of further research of doubly heavy baryon states: their decays, productions and possibility of observation of excited states.

The rest of the paper is organized as follows. In the next section production of doubly heavy baryons is considered. Section III is devoted to theoretical calculation of the lifetimes of the considered particles. Observation probability of these baryons is discussed in Sec. IV and finally the Conclusion will be given.

\section{DOUBLY HEAVY BARYON PRODUCTION}

It is natural to use a two-step procedure to produce a doubly heavy baryon. In the first calculation step a doubly heavy diquark is produced perturbatively in the hard interaction. In the second step a doubly heavy diquark is transformed to the baryon within the soft hadronization process.

Our calculation of doubly heavy diquark production was done within the following approach:

(1) the color singlet model for doubly heavy mesons and the color triplet model for doubly heavy baryons;

\footnotetext{
${ }^{1}$ An alternative approach based on the direct solution of the three-body problem is presented in $[2,3]$.
} 

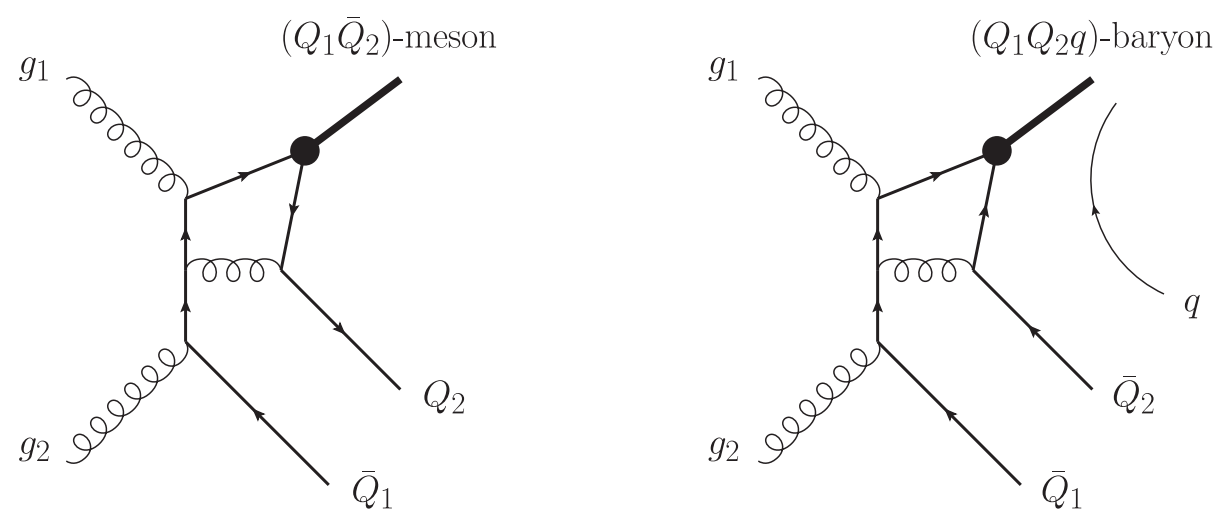

FIG. 1. The example of analogous diagrams for $\left(Q_{1} \bar{Q}_{2}\right)$-quarkonium production and for $\left(Q_{1} Q_{2} q\right)$-baryon production.

(2) the contribution from scattering of sea heavy quark and gluon $\left(Q_{1} g \rightarrow Q_{1}+Q_{2}+\bar{Q}_{2}\right)$ does not take into account to avoid double counting ${ }^{2}$;

(3) the contribution of color sextet state to baryon production is neglected.

Quarks in color antitriplet $\overline{3}_{c}$ attract each other and their interaction can be described by the wave function in the framework of the potential model, as well as the quarkantiquark interaction in quarkonium. By analogy with quarkonium one can write for the production amplitude of the doubly heavy diquark

$A^{S J j_{z}}=\int T_{Q_{1} \bar{Q}_{1} Q_{2} \bar{Q}_{2}}^{S s_{z_{i}}}\left(p_{i}, k(\vec{q})\right) \cdot\left(\Psi_{\left[Q_{1} Q_{2}\right]_{\overline{3}_{c}}}^{L l_{\bar{s}_{z}}}(\vec{q})\right)^{*} \cdot C_{s_{z} l_{z}}^{J j_{z}} \frac{d^{3} \vec{q}}{(2 \pi)^{3}}$,

where $T_{Q_{1} \bar{Q}_{1} Q_{2} \bar{Q}_{2}}^{S s_{z}}$ is an amplitude of the hard production of two heavy quark pairs; $\Psi_{\left[Q_{1} Q_{2}\right]_{\bar{s}_{c}}}^{L l_{z}}$ is the diquark wave function (color antitriplet); $J$ and $j_{z}$ are the total angular momentum and its projection on the $z$-axis in the $\left[Q_{1} Q_{2}\right]_{\overline{3}_{c}}$ rest frame; $L$ and $l_{z}$ are the orbital angular momentum of a $b c$ diquark and its projection on the $z$-axis; $S$ and $s_{z}$ are the $Q_{1} Q_{2}$-diquark spin and its projection; $C_{s_{z} l_{z}}^{J_{z}}$ are ClebschGordan coefficients; $p_{i}$ are the four-momenta of the diquark, $\bar{Q}_{1}$ quark and $\bar{Q}_{2}$ quark; and $\vec{q}$ is the threemomentum of the $Q_{1}$ quark in the $Q_{1} Q_{2}$-diquark rest frame [in this frame $(0, \vec{q})=k(\vec{q})$ ].

Under the assumption of small dependence of $T_{b \bar{b} c \bar{c}}^{S S_{z}}$ on $k(\vec{q})$

$$
A \sim \int d^{3} q \Psi^{*}(\vec{q})\left\{\left.T\left(p_{i}, \vec{q}\right)\right|_{\vec{q}=0}+\left.\vec{q} \frac{\partial}{\partial \vec{q}} T\left(p_{i}, \vec{q}\right)\right|_{\vec{q}=0}+\cdots\right\}
$$

and, particularly, for the $S$-wave states

\footnotetext{
${ }^{2}$ Furthermore, accounting these process is questionable for $\mathrm{LHCb}$ kinematic region due to rather small transverse momenta of a doubly heavy system which is comparable with a heavy quark mass.
}

$$
\left.A \sim R_{S}(0) \cdot T_{Q_{1} \bar{Q}_{1} Q_{2} \bar{Q}_{2}}\left(p_{i}\right)\right|_{\vec{q}=0}
$$

where $R_{S}(0)$ is a value of the radial wave function at the origin.

In our early work [6] we discussed the similarity of the production mechanisms of doubly charmed baryons and the associative $J / \psi$ and the open charm in hadronic interactions. Indeed, both processes within a single parton scattering approach are described by the similar sets of diagrams, because both involve the production of four heavy quarks (see the diagram examples in Fig. 1). However, the experimental data indicate the presence of thecontribution of double parton scattering (DPS), which dominates at LHC energies [7]. Within the DPS mechanism two $c \bar{c}$ pairs are produced independently in the different parton interactions. Such a mechanism can contribute to the associative $J / \psi+c$ production but one can hardly contribute to the process $\Xi_{c c}$ production, because charm quarks from different pairs are needed to produce doubly charmed baryon. ${ }^{3}$ Thus we currently tend to think that the DPS mechanism contributes only to $J / \psi+c$ production. This is why the yield of $\Xi_{c c}$ is essentially smaller than the yield of the associative production of the $J / \psi$ meson and open charm, whereas the yields of $B_{c}$ mesons and $\Xi_{b c}$ baryons should be comparable. Also it is worth mentioning that the $J / \psi+c$ cross section and $\Xi_{c c}$ cross section should have different dependence on the $p p$ interaction energy: the DPS cross section increases faster than SPS.

It should be noted that the doubly heavy diquark production cannot be described within the fragmentation model due to the large contribution of nonfragmentation diagrams, which cannot be interpreted as $b$-quark production followed by the fusion of $b$ quarks into $b c$ diquarks. The same feature is inherent in the process of $B_{c}$-meson production. This is not surprising because the production processes of $b c$-diquark production and $B_{c}$ production are

\footnotetext{
${ }^{3}$ However there is a research work where an attempt was made to expand the DPS model to the case of $\Xi_{c c}$ production [8] using the quark-hadron duality approach.
} 

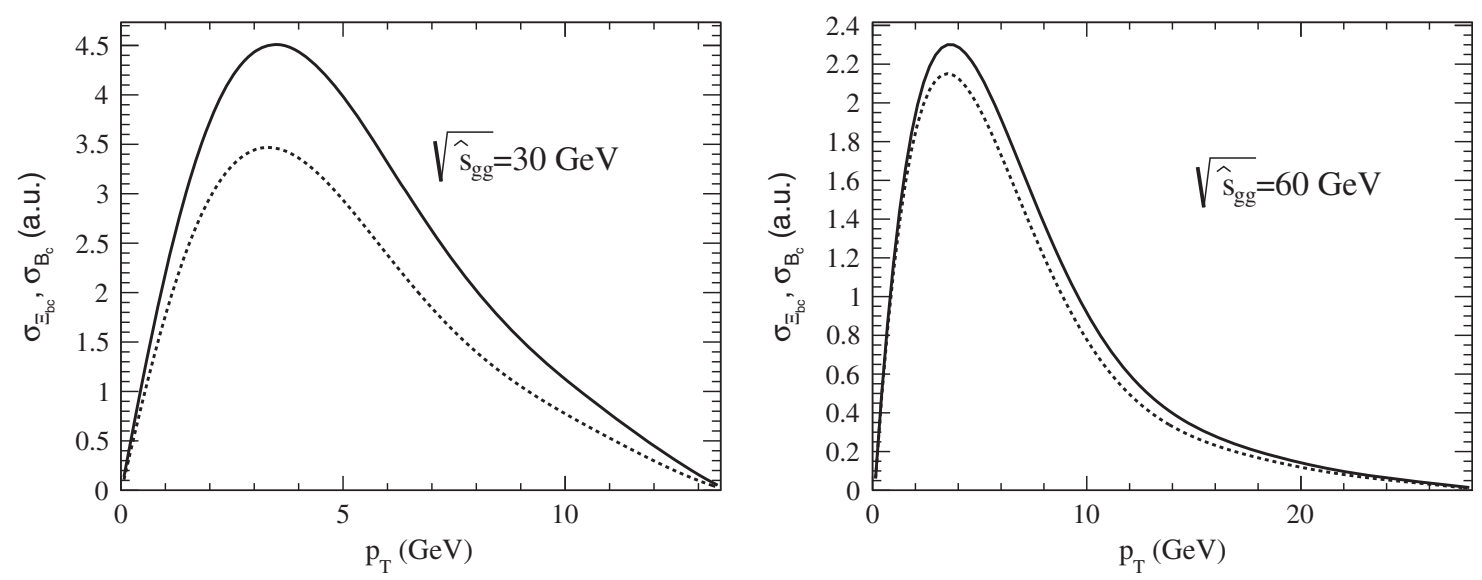

FIG. 2. $\Xi_{b c} p_{T}$ distribution vs. $B_{c} p_{T}$ distribution for $\sqrt{s_{g g}}=30 \mathrm{GeV}$ and $\sqrt{s_{g g}}=60 \mathrm{GeV}$, respectively. The same quark mass values are used for both estimations: $m_{c}=1.5 \mathrm{GeV}$ and $m_{b}=4.8 \mathrm{GeV}$. Also, for the convenience of comparison, we put $\left|R_{B_{c}}(0)\right|^{2}$ and $\left|R_{[b c]_{3}}(0)\right|^{2}$ equal.

described by the same set of the diagrams. The difference comes from different color coefficients and different choices of values for the $c$ and $b$ quark masses.

The dominant contribution to the production cross under $\mathrm{LHCb}$ kinematics conditions comes from gluonic interaction, as well as from the $B_{c}$ meson:

$$
g g \rightarrow \Xi_{b c}+\bar{b} \bar{c}
$$

Our estimations for that process show that the difference of yields of $\Xi_{b c}$ and $B_{c}$ is mostly determined by the difference of wave functions:

$$
\frac{\sigma_{\Xi_{b c}}}{\sigma_{B_{c}}} \sim \frac{\left|R_{[b c]}(0)\right|^{2}}{\left|R_{B_{c}}(0)\right|^{2}} .
$$

Indeed, if one chooses the same quark mass values for the subprocesses $g g \rightarrow[b c]_{\overline{3}}+\bar{b} \bar{c}$ and $g g \rightarrow B_{c}+\bar{b} \bar{c}$ and puts $R_{[b c]_{\overline{3}}}^{2}=R_{B_{c}}^{2}$ one can see that this process has very similar behavior on the transverse momenta of a doubly heavy system, as it is shown in Fig. 2, where we put $\left|R_{B_{c}}(0)\right|^{2}$ and $\left|R_{[b c]_{\overline{3}}}(0)\right|^{2}$ equal for convenience of comparison.

Of course, a color antitriplet of the $b c$ system is not a $\Xi_{b c}$ yet. It should be somehow transformed to the $b c q$ baryon. The transverse momentum of light quark $q$ with mass $m_{q}$ is about $\frac{m_{q}}{m_{\Xi_{b c}}} p_{T}^{\Xi_{b c}}$, where $p_{T}^{\Xi_{b c}}$ is a transverse momentum of $\Xi_{b c}$. For LHCb kinematical conditions such a quark always exits in the quark sea. This is why we assume that a doubly heavy is hadronized by joining with the light quarks $u, d$ and $s$ in the proportion $1: 1: 0.3$. We also assume that it is hadronized with probability equal 1 . It is worth noting that the latter assumption is pretty much a guess, because the diquark has a color charge and therefore strongly interacts with its environment, which could lead to the diquark dissociation. Thus, (3) can be considered as an upper limit for the ratio of yields of $\Xi_{b c}$ and $B_{c}$.

We estimate the ratio of yields $\Xi_{b c}$ and $B_{c}$ for hadronic interactions at $\sqrt{s}=13 \mathrm{TeV}$ for several scales $\left(\mu_{R}=\mu_{F}=\right.$ $10 \mathrm{GeV}, \mu_{R}=\mu_{F}=E_{T}^{\Xi_{b c}} / 2, \mu_{R}=\mu_{F}=E_{T}^{\Xi_{b c}}, \mu_{R}=\mu_{F}=$ $\left.2 E_{T}^{\Xi_{b c}}\right)$ and find that the dependence of this value on scale choice is unessential. The main uncertainties come from wave functions and from the choice of mass values for $b$ and $c$ quarks. In Fig. 3 we show the ratio of yields $\Xi_{b c}$ and $B_{c}$ in hadronic interactions as a function of $p_{T}$ at $\sqrt{s}=13 \mathrm{TeV}$, for similar masses $\left(m_{b}=4.8 \mathrm{GeV}, m_{c}=1.5 \mathrm{GeV}\right)$ and for different masses $\left(m_{b}=4.8 \mathrm{GeV}\right.$ and $m_{c}=1.5 \mathrm{GeV}$ for $B_{c}$

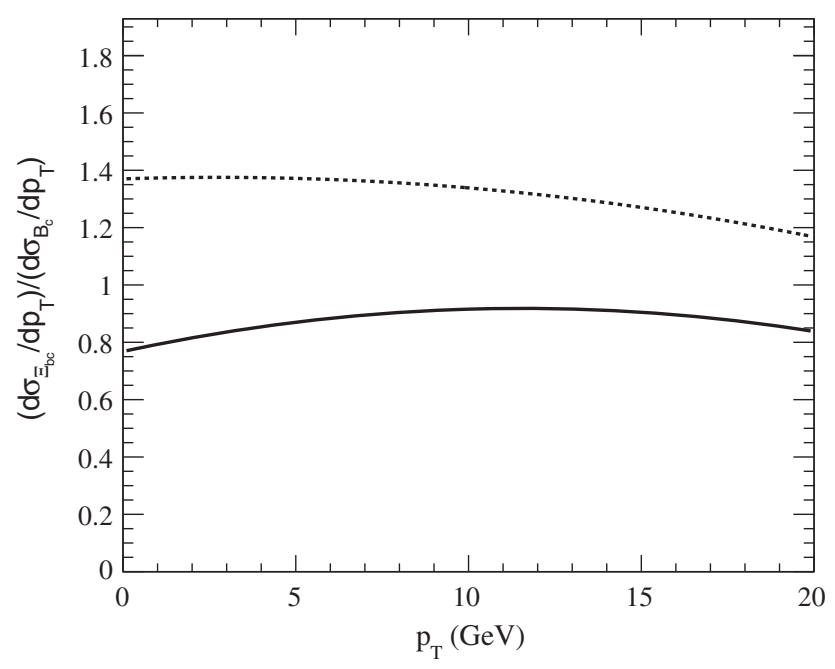

FIG. 3. The ratio of production yields of $\Xi_{b c}$ and $B_{c}$ for hadronic interaction at $\sqrt{s}=13 \mathrm{TeV}$ in units of $\left|R_{[b c]_{\overline{3}}}(0)\right|^{2} /\left|R_{B_{c}}(0)\right|^{2}$ for the similar quark masses $\left[m_{b}=4.8 \mathrm{GeV}, m_{c}=1.5 \mathrm{GeV}\right.$ (solid curve) $]$ and for the different quark masses $\left[m_{b}=4.8 \mathrm{GeV}\right.$ and $m_{c}=1.5 \mathrm{GeV}$ for $B_{c}$ production, and $m_{b}=4.9 \mathrm{GeV}$ and $m_{c}=$ $1.7 \mathrm{GeV}$ for $\Xi_{b c}$ production (dashed curve)]. The CT14LL parametrization [9] is used for PDFs. 
production, and $m_{b}=4.9 \mathrm{GeV}$ and $m_{c}=1.7 \mathrm{GeV}$ for $\Xi_{b c}$ production). Here we also put $\left|R_{B_{c}}(0)\right|^{2}=\left|R_{[b c]_{\overline{3}}}(0)\right|^{2}$. One can see that these distributions are approximately flat. Thus, one can conclude that the estimation (3) is approximately valid for all transverse momenta.

There are many estimations for the $R_{[b c]_{3}}(0)$ value, as well as for $R_{B_{c}}(0)$ (see, for example, [1,10-13]). However, to obtain the ratio, it is rational to use values extracted within the similar framework. From [1,10], where the nonrelativistic model with a Buchmüller-Tye wave function was used, we obtain that

$$
\frac{\left|R_{[b c]}(0)\right|^{2}}{\left|R_{B_{c}}(0)\right|^{2}}=\frac{\left(0.71 \mathrm{GeV}^{3 / 2}\right)^{2}}{\left(1.28 \mathrm{GeV}^{3 / 2}\right)^{2}} \approx 0.31 .
$$

From [12,11], where the relativistic potential model was applied and relativistic corrections have been accounted for perturbatively, we obtain for the same ratio

$$
\frac{\left|R_{[b c]}(0)\right|^{2}}{\left|R_{B_{c}}(0)\right|^{2}}=\frac{\left(0.74 \mathrm{GeV}^{3 / 2}\right)^{2}}{\left(1.46 \mathrm{GeV}^{3 / 2}\right)^{2}} \approx 0.26 .
$$

In $[14,15]$ the corrections to the relativistic potential model predictions were taken into account nonperturbatively, which leads to a noticeable difference in the wave function values for different spin states. However the cross section ratio value remains the same:

$$
\begin{aligned}
\frac{\sigma_{\Xi_{b c}}}{\sigma_{B_{c}}} & =\frac{\sigma_{\Xi_{b c}\left(1^{1} S_{0}\right)}+\sigma_{\Xi_{b c}\left(1^{3} S_{1}\right)}}{\sigma_{B_{c}\left(1^{1} S_{0}\right)}+\sigma_{B_{c}\left(1^{3} S_{1}\right)}} \\
& \approx \frac{\left|R_{[b c]_{3}\left(1^{1} S_{0}\right)}(0)\right|^{2}+3 \cdot\left|R_{[b c]_{3}\left(1^{3} S_{1}\right)}(0)\right|^{2}}{\left|R_{B_{c}\left(1^{1} S_{0}\right)}(0)\right|^{2}+2.5 \cdot\left|R_{B_{c}\left(1^{3} S_{1}\right)}(0)\right|^{2}} \\
& \approx \frac{\left(0.84 \mathrm{GeV}^{3 / 2}\right)^{2}+3 \cdot\left(0.59 \mathrm{GeV}^{3 / 2}\right)^{2}}{\left(1.64 \mathrm{GeV}^{3 / 2}\right)^{2}+2.5 \cdot\left(1.05 \mathrm{GeV}^{3 / 2}\right)^{2}} \approx 0.32 .
\end{aligned}
$$

Therefore, one can conclude that

$$
\frac{\sigma_{\Xi_{b c}}}{\sigma_{B_{c}}} \lesssim \frac{1}{3} .
$$

It is worth noting that both the numerator and the denominator in (4) will be modified by the feed-down from excitations. However we believe that in a ratio these contributions will approximately cancel out. The obtained ratio value $\sigma_{\Xi_{b c}} / \sigma_{B_{c}}$ coincides with that used in the talk in [16].

To estimate the absolute cross section value of $\Xi_{b c}$ baryon production at $\mathrm{LHCb}\left(\sqrt{s}=13 \mathrm{TeV}, 2.0<y_{\Xi_{b c}}<4.5\right)$ we use the quark mass values $m_{b}=4.9 \mathrm{GeV}$ and $m_{c}=1.7 \mathrm{GeV}$, the value of the diquark wave function at the origin $R_{[b c]_{3}}(0)=0.71 \mathrm{GeV}^{3 / 2}$ [1] and CT14LL parton density parametrization [9]. Varying scales from $\mu_{R}=\mu_{F}=E_{T}^{\Xi_{b c}} / 2$ to $\mu_{R}=\mu_{F}=2 E_{T}^{\Xi_{b c}}$ we obtain that the cross section value of $b c$ baryons with a $1 S$ wave state of a doubly heavy diquark at $\mathrm{LHCb}$ is about $10-25 \mathrm{nb}$ depending on scale values. The feed-down from excitations can be estimated as $20 \%-30 \%$.

As mentioned before an analogous ratio cannot be valid for $J / \psi+c$ and $\Xi_{c c}$ due to the large contribution of DPS to the associative $J / \psi$ and $c$ production.

\section{DOUBLY HEAVY BARYON DECAYS WITHIN OPE METHOD}

\section{A. Method description}

In accordance with operator product expansion (OPE) and the optic theorem the lifetime of a doubly heavy baryon $\mathcal{B}$ can be represented as

$$
\Gamma_{\mathcal{B}}=\frac{1}{2 M_{\mathcal{B}}}\langle\mathcal{B}|\mathcal{T}| \mathcal{B}\rangle,
$$

where operator $\mathcal{T}$ is

$$
\mathcal{T}=\operatorname{Im} \int d^{4} x\left\{\hat{T} H_{\mathrm{eff}}(x) H_{\mathrm{eff}}(0)\right\}
$$

with

$$
H_{\text {eff }}=\frac{G_{F}}{2 \sqrt{2}} V_{q_{3} q_{4}} V_{q_{1} q_{2}}^{*}\left[C_{+}(\mu) O_{+}+C_{-}(\mu) O_{-}\right] .
$$

In the above expression the Wilson coefficients $C_{ \pm}(\mu)$ equal

$C_{+}(\mu)=\left[\frac{\alpha_{s}\left(M_{W}\right)}{\alpha_{s}(\mu)}\right]^{\frac{6}{33-2 n_{f}}}, \quad C_{-}(\mu)=\left[\frac{\alpha_{s}\left(M_{W}\right)}{\alpha_{s}(\mu)}\right]^{-\frac{12}{33-2 n_{f}}}$,

where $\alpha_{s}(\mu)$ is a running strong coupling constant calculated within the two-loop approximation and $n_{f}$ is a number of active flavors. The operators $O_{ \pm}$in (7) are determined as follows:

$O_{ \pm}=\left[\bar{q}_{1 \alpha} \gamma_{\nu}\left(1-\gamma_{5}\right) q_{2 \beta}\right]\left[\bar{q}_{3 \gamma} \gamma^{\nu}\left(1-\gamma_{5}\right) q_{4 \delta}\right]\left(\delta_{\alpha \beta} \delta_{\gamma \delta} \pm \delta_{\alpha \delta} \delta_{\beta \gamma}\right)$,

where $\alpha, \beta, \gamma$, and $\delta$ are the color indices of quarks.

For a large energy of heavy quark decay one can represent $\mathcal{T}(6)$ as a set of local operators ordered by increasing dimension. The contributions of high dimension terms are suppressed by inverse powers of heavy quark mass $m_{Q}$, and therefore only several first terms contribute to the decay value. This method was broadly used for the calculation of lifetimes of heavy hadrons [6,17-23], as well as doubly heavy hadrons [24,25]. It was shown in the cited papers that the operators of dimensions 3 and 5

$$
O_{Q Q}=(\bar{Q} Q), \quad O_{Q G}=\left(\bar{Q} \sigma_{\mu \nu} G^{\mu \nu} Q\right),
$$

correspond to the spectator decay of a heavy quark and give the main contribution to the value (5). The following 


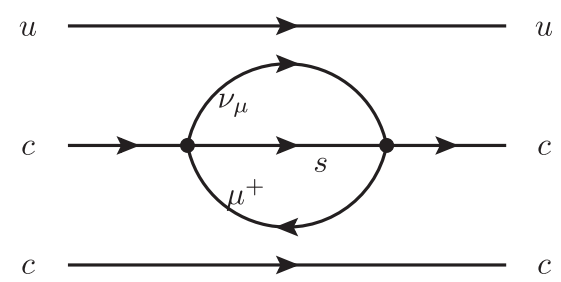

(a)

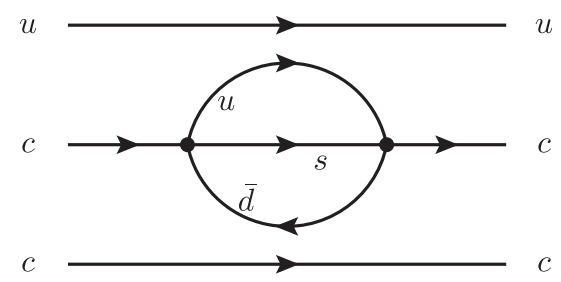

(c)

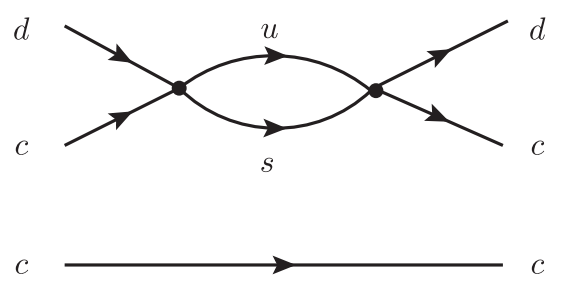

(b)

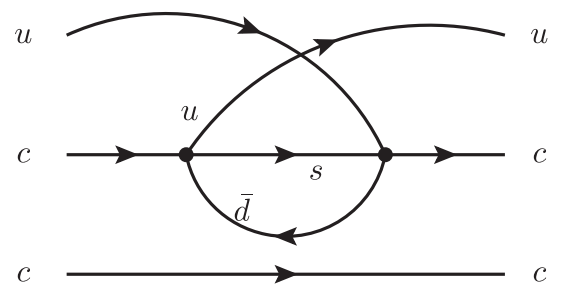

(d)

FIG. 4. Feynman diagrams for $\Xi_{c c}$ baryon decay: (a) spectator mechanism, (b) weak scattering and (c,d) Pauli interference.

operator of dimension 6 can also give a noticeable contribution to the decay process:

$$
O_{2 Q 2 q}=(\bar{Q} \Gamma q)(\bar{q} \gamma Q)
$$

The other operators of dimension 6, $O_{61 Q}=\bar{Q} \sigma_{\mu \nu} \gamma_{\lambda} D^{\mu} G^{\nu \lambda} Q$, $O_{62 Q}=\bar{Q} D_{\mu} G^{\mu \nu} \Gamma_{\nu} Q$, contribute insignificantly compared with (11).

Typical Feynman diagrams for the discussed processes are shown in Fig. 4. In accordance with the OPE method the following mechanisms can contribute to the total decay width:

(i) Spectator mechanism [the operator (10) and the diagram 4(a)],

(ii) Weak scattering (WS) [the operator (11) and the diagram 4(b)],

(iii) Pauli interference (PI) [the operator (11) and the diagrams 4(c) and 4(d)].
B. Lifetimes of doubly charmed baryons $\Xi_{c c}^{++}, \Xi_{c c}^{+}, \Omega_{c c}^{+}$

The decay amplitudes for doubly charmed baryons $\Xi_{c c}^{++}$ and $\Xi_{c c}^{+}$can be performed as follows:

$$
\begin{aligned}
& \mathcal{T}_{\Xi_{c c}^{++}}=2 \mathcal{T}_{35 c}+\mathcal{T}_{\mathrm{PI}}^{\left(\Xi_{c c}^{++}\right)}, \\
& \mathcal{T}_{\Xi_{c c}^{+}}=2 \mathcal{T}_{35 c}+\mathcal{T}_{\mathrm{WS}}^{\left(\Xi_{c c}^{+}\right)} \\
& \mathcal{T}_{\Omega_{c c}^{+}}=2 \mathcal{T}_{35 c}+\mathcal{T}_{\mathrm{PI}}^{\left(\Omega_{c c}^{+}\right)} .
\end{aligned}
$$

In these equations the contribution of operators with dimensions 3 and 5 can be determined as follows:

$$
\mathcal{T}_{35 c}=\Gamma_{c, \mathrm{spec}}(\bar{c} c)-\frac{\Gamma_{0 c}}{m_{c}^{2}}\left[\left(2+K_{0 c}\right) P_{s 1}+K_{2 c} P_{s 2}\right] O_{G c}
$$

where

$$
\begin{aligned}
\Gamma_{0 c}= & \frac{G_{F}^{2} m_{c}^{5}}{192 \pi^{3}}, \quad K_{0 Q}=C_{-}^{2}+2 C_{+}^{2}, \quad K_{2 Q}=2\left(C_{+}^{2}-C_{-}^{2}\right) \\
P_{c 1}= & (1-y)^{4}, \quad P_{c 2}=(1-y)^{3}, \quad y=\frac{m_{s}^{2}}{m_{c}^{2}}, \quad r=\frac{m_{\tau}^{2}}{m_{c}^{2}} \\
P_{c \tau 1}= & \sqrt{1-2(r+y)+(r-y)^{2}}\left[1-3(r+y)+3\left(r^{2}+y^{2}\right)-r^{3}-y^{3}\right. \\
& -4 r y+7 r y(r+y)]+12 r^{2} y^{2} \ln \frac{\left(1-r-y+\sqrt{1-2(r+y)+(r-y)^{2}}\right)^{2}}{4 r y}, \\
P_{c c 1}= & \sqrt{1-4 y}\left(1-6 y+2 y^{2}+12 y^{3}\right) 24 y^{4} \ln \frac{1+\sqrt{1-4 y}}{1-\sqrt{1-4 y}} \\
P_{c c 2}= & \sqrt{1-4 y}\left(1+\frac{y}{2}+3 y^{2}\right)-3 y\left(1-2 y^{2}\right) \ln \frac{1+\sqrt{1-4 y}}{1-\sqrt{1-4 y}},
\end{aligned}
$$


and the width of the spectator mechanism was estimated in the papers [24,26-31].

As mentioned above the contribution values of the PI and WS mechanisms depend on the baryon composition. For example, it is clear from diagrams in Fig. 4 that for $\Xi_{c c}^{++}=$ (ccu) and $\Omega_{c c}^{+}=(c c s)$ the WS is forbidden and PI destructively contributes to the width. In contrast, for the $\Xi_{c c}^{+}$the PI is forbidden. Keeping this in mind one can perform the contributions of operators of dimension 6 as follows:

where (see, e.g., [24,32-34])

$$
\begin{aligned}
& \mathcal{T}_{\mathrm{PI}, u \bar{d}}^{c}=-\frac{G_{F}^{2}}{4 \pi} m_{c}^{2}\left(1-\frac{m_{u}}{m_{c}}\right)^{2}\left\{\left[G_{1}\left(z_{-}\right)(\bar{c} c)_{V-A}^{i i}(\bar{u} u)_{V-A}^{j j}+G_{2}\left(z_{-}\right)(\bar{c} c)_{A}^{i i}(\bar{u} u)_{V-A}^{j j}\right]\left[F_{3}+\frac{1}{3}\left(1-k^{\frac{1}{2}}\right) F_{4}\right]+\right. \\
&\left.\times\left[G_{1}\left(z_{-}\right)(\bar{c} c)_{V-A}^{i j}(\bar{u} u)_{V-A}^{j i}+G_{2}\left(z_{-}\right)(\bar{c} c)_{A}^{i j}(\bar{u} u)_{V-A}^{j i}\right] k^{\frac{1}{2}} F_{4}\right\}, \\
& \mathcal{T}_{\mathrm{WS}, c d}= \frac{G_{F}^{2}}{4 \pi} m_{c}^{2}\left(1+\frac{m_{d}}{m_{c}}\right)^{2}\left(1-z_{+}\right)^{2}\left[\left(F_{6}+\frac{1}{3}\left(1-k^{\frac{1}{2}}\right) F_{5}\right)(\bar{c} c)_{V-A}^{i i}(\bar{d} d)_{V-A}^{j j}+k^{\frac{1}{2}} F_{5}(\bar{c} c)_{V-A}^{i j}(\bar{d} d)_{V-A}^{j i}\right], \\
& \mathcal{T}_{\mathrm{PI}, u \bar{d}}^{c^{\prime}}=-\frac{G_{F}^{2}}{4 \pi} m_{c}^{2}\left(1-\frac{m_{s}}{m_{c}}\right)^{2}\left\{\left[\frac{1}{4}(\bar{c} c)_{V-A}^{i i}(\bar{s} s)_{V-A}^{j j}+\frac{1}{6}(\bar{c} c)_{A}^{i i}(\bar{s} s)_{V-A}^{j j}\right]\left[F_{1}+\frac{1}{3}\left(1-k^{\frac{1}{2}}\right) F_{2}\right]\right. \\
&\left.+\left[\frac{1}{4}(\bar{c} c)_{V-A}^{i j}(\bar{s} s)_{V-A}^{j i}+\frac{1}{6}(\bar{c} c)_{A}^{i j}(\bar{s} s)_{V-A}^{j i}\right] k^{\frac{1}{2}} F_{2}\right\}, \\
& \mathcal{T}_{\mathrm{PI}, \nu_{\tau} \bar{\tau}}^{c}=-\frac{G_{F}^{2}}{\pi} m_{c}^{2}\left(1-\frac{m_{s}}{m_{c}}\right)^{2}\left[G_{1}\left(z_{\tau}\right)(\bar{c} c)_{V-A}^{i j}(\bar{s} s)_{V-A}^{j i}+G_{2}\left(z_{\tau}\right)(\bar{c} c)_{A}^{i j}(\bar{s} s)_{V-A}^{j i}\right], \\
& \mathcal{T}_{\mathrm{P} \mathrm{I}, \nu_{e} \bar{e}}^{c}=\mathcal{T}_{\mathrm{P} \mathrm{I}, \nu_{\mu} \bar{\mu}}^{c}=\mathcal{T}_{\mathrm{PI}, \nu_{\tau} \bar{\tau}}^{c}\left(z_{\tau} \rightarrow 0\right)
\end{aligned}
$$

and

$$
\begin{aligned}
& (13): z_{-}=\frac{m_{s}^{2}}{\left(m_{c}-m_{u}\right)^{2}}, k=\frac{\alpha_{s}(\mu)}{\alpha_{s}\left(m_{c}-m_{u}\right)}, \\
& (14): z_{+}=\frac{m_{s}^{2}}{\left(m_{c}+m_{d}\right)^{2}}, k=\frac{\alpha_{s}(\mu)}{\alpha_{s}\left(m_{c}+m_{d}\right)} . \\
& (15): k=\frac{\alpha_{s}(\mu)}{\alpha_{s}\left(m_{c}-m_{s}\right)} . \\
& (16): z_{\tau}=\frac{m_{\tau}^{2}}{\left(m_{c}-m_{s}\right)^{2}} .
\end{aligned}
$$

In these relations we also introduce the notations

$$
\begin{aligned}
F_{1,3} & =\left(C_{+} \mp C_{-}\right)^{2}, \quad F_{2,4}=5 C_{+}^{2}+C_{-}^{2} \pm 6 C_{+} C_{-}, \quad F_{5,6}=C_{+}^{2} \mp C_{-}^{2} \\
G_{1}(z) & =\frac{(1-z)^{2}}{2}-\frac{(1-z)^{3}}{4}, \quad G_{2}(z)=\frac{(1-z)^{2}}{2}-\frac{(1-z)^{3}}{3}, \\
(\bar{q} q)_{A}^{i j} & =\left(\bar{q}^{i} \gamma_{\alpha} \gamma_{5} q^{j}\right), \quad(\bar{q} q)_{V-A}^{i j}=\left(\bar{q}^{i} \gamma_{\alpha}\left(1-\gamma_{5}\right) q^{j}\right) .
\end{aligned}
$$

The hadronic matrix elements are determined as follows: 


$$
\begin{aligned}
\left\langle\Xi_{Q Q}^{\diamond}\left|\left(\bar{Q}_{\gamma_{\mu}}\left(1-\gamma_{5}\right) Q\right)\left(\bar{q} \gamma^{\mu}\left(1-\gamma_{5}\right) q\right)\right| \Xi_{Q Q}^{\diamond}\right\rangle & =12\left(m_{Q}+m_{q}\right) \cdot\left|\Psi^{d l}(0)\right|^{2}, \\
\left\langle\Xi_{Q Q}^{\diamond}\left|\left(\bar{Q} \gamma_{\mu} \gamma_{5} Q\right)\left(\bar{q} \gamma^{\mu}\left(1-\gamma_{5}\right) q\right)\right| \Xi_{Q Q}^{\diamond}\right\rangle & =8\left(m_{Q}+m_{q}\right) \cdot\left|\Psi^{d l}(0)\right|^{2}, \\
\left\langle\Omega_{Q Q}\left|\left(\bar{Q}_{\gamma_{\mu}}\left(1-\gamma_{5}\right) Q\right)\left(\bar{s} \gamma^{\mu}\left(1-\gamma_{5}\right) s\right)\right| \Omega_{Q Q}\right\rangle & =12\left(m_{Q}+m_{s}\right) \cdot\left|\Psi^{d l}(0)\right|^{2}, \\
\left\langle\Omega_{Q Q}\left|\left(\bar{Q}_{\gamma_{\mu}} \gamma_{5} Q\right)\left(\bar{s} \gamma^{\mu}\left(1-\gamma_{5}\right) s\right)\right| \Omega_{Q Q}\right\rangle & =8\left(m_{Q}+m_{s}\right) \cdot\left|\Psi^{d l}(0)\right|^{2},
\end{aligned}
$$

where $Q=c, b$ is the heavy quark; $q=u, d$ is the light quark; and $\left|\Psi^{d l}(0)\right|^{2}$ is a wave function at the origin. The wave function structure leads to the following relation:

$$
\left\langle\Xi_{Q Q^{\prime}}^{\diamond}\left|\left(\bar{Q}_{i} T_{\mu} Q_{k}\right)\left(\bar{q}_{k} \gamma^{\mu}\left(1-\gamma_{5}\right) q_{i}\right)\right| \Xi_{Q Q^{\prime}}^{\diamond}\right\rangle=-\left\langle\Xi_{Q Q^{\prime}}^{\diamond}\left|\left(\bar{Q} T_{\mu} Q\right)\left(\bar{q} \gamma^{\mu}\left(1-\gamma_{5}\right) q\right)\right| \Xi_{Q Q^{\prime}}^{\diamond}\right\rangle
$$

where $T_{\mu}$ is an arbitrary spinor matrix.

\section{Lifetimes of doubly beauty baryons $\Xi_{b b}^{0}, \Xi_{b b}^{-}, \Omega_{b b}^{-}$}

For the double beauty baryons $\Xi_{b b}^{0}=(b b u), \Xi_{b b}^{-}=$ $(b b d)$ and $\Omega_{b b}^{-}=(b b s)$ the WS mechanism contributes only to the width of neutral states, whereas for the charged states the PI mechanism contribution must be accounted for:

$$
\begin{aligned}
& \mathcal{T}_{\Xi_{b b}^{0}}=2 \mathcal{T}_{35 b}+\mathcal{T}_{\mathrm{WS}}^{\left(\Xi_{b b}^{0}\right)}, \\
& \mathcal{T}_{\Xi_{b b}^{-}}=2 \mathcal{T}_{35 b}+\mathcal{T}_{\mathrm{PI}}^{\left(\Xi_{b b}^{-}\right)}, \\
& \mathcal{T}_{\Omega_{b b}^{-}}=2 \mathcal{T}_{35 b}+\mathcal{T}_{\mathrm{PI}}^{\left(\Omega_{b b}^{-}\right)} .
\end{aligned}
$$

The spectator mechanism of $b$-quark decay is described by the following operators with dimensions 3 and 5:

$$
\begin{aligned}
\mathcal{T}_{35 b}= & \Gamma_{b, \text { spec }}(\bar{b} b)-\frac{\Gamma_{0 b}}{m_{b}^{2}}\left[2 P_{c 1}+P_{c \tau 1}+K_{0 b}\left(P_{c 1}+P_{c c 1}\right)\right. \\
& +K_{2 b}\left(P_{c 2}+P_{c c 2}\right] O_{G b},
\end{aligned}
$$

where

$$
\Gamma_{0 c}=\frac{G_{F}^{2} m_{c}^{5}}{192 \pi^{3}},
$$

and the other functions are determined earlier. The operators of dimension 6 equal

$$
\mathcal{T}_{\mathrm{WS}}^{\left(\Xi_{b b}^{0}\right)}=2 \mathcal{T}_{\mathrm{WS}, b u}, \quad \mathcal{T}_{\mathrm{PI}}^{\left(\Xi_{b b}^{-}\right)}=2 \mathcal{T}_{\mathrm{PI}, d \bar{u}}^{b^{\prime}}, \quad \mathcal{T}_{\mathrm{PI}}^{\left(\Omega_{b b}^{-}\right)}=2 \mathcal{T}_{\mathrm{PI}, s \bar{c}}^{b^{\prime}},
$$

where [35]

$$
\begin{aligned}
\mathcal{T}_{\mathrm{WS}, b u}= & \frac{G_{F}^{2}\left|V_{c b}\right|^{2}}{4 \pi} m_{b}^{2}\left(1+\frac{m_{u}}{m_{b}}\right)^{2}\left(1-z_{+}\right)^{2}\left[\left(F_{6}+\frac{1}{3}\left(1-k^{\frac{1}{2}}\right) F_{5}\right)(\bar{b} b)_{V-A}^{i i}(\bar{u} u)_{V-A}^{j j}+k^{\frac{1}{2}} F_{5}(\bar{b} b)_{V-A}^{i j}(\bar{u} u)_{V-A}^{j i}\right], \\
\mathcal{T}_{\mathrm{PI}, d \bar{u}}^{b^{\prime}}= & -\frac{G_{F}^{2}\left|V_{c b}\right|^{2}}{4 \pi} m_{b}^{2}\left(1-\frac{m_{d}}{m_{b}}\right)^{2}\left\{\left[G_{1}\left(z_{-}\right)(\bar{b} b)_{V-A}^{i i}(\bar{d} d)_{V-A}^{j j}+G_{2}\left(z_{-}\right)(\bar{b} b)_{A}^{i i}(\bar{d} d)_{V-A}^{j j}\right]\right. \\
& \left.\times\left[F_{3}+\frac{1}{3}\left(1-k^{\frac{1}{2}}\right) F_{4}\right]+\left[G_{1}\left(z_{-}\right)(\bar{b} b)_{V-A}^{i j}(\bar{d} d)_{V-A}^{j i}+G_{2}\left(z_{-}\right)(\bar{b} b)_{A}^{i j}(\bar{d} d)_{V-A}^{j i}\right] k^{\frac{1}{2}} F_{4}\right\}, \\
\mathcal{T}_{\mathrm{PI}, s \bar{c}}^{b^{\prime}}= & -\frac{G_{F}^{2}\left|V_{c b}\right|^{2}}{16 \pi} m_{b}^{2}\left(1-\frac{m_{s}}{m_{b}}\right)^{2} \sqrt{\left(1-4 z_{-}\right)}\left\{\left[\left(1-z_{-}\right)(\bar{b} b)_{V-A}^{i i}(\bar{s} s)_{V-A}^{j j}+\frac{2}{3}\left(1+2 z_{-}\right)(\bar{b} b)_{A}^{i i}(\bar{s} s)_{V-A}^{j j}\right]\right. \\
& \left.\times\left[F_{3}+\frac{1}{3}\left(1-k^{\frac{1}{2}}\right) F_{4}\right]+\left[\left(1-z_{-}\right)(\bar{b} b)_{V-A}^{i j}(\bar{s} s)_{V-A}^{j i}+\frac{2}{3}\left(1+2 z_{-}\right)(\bar{b} b)_{A}^{i j}(\bar{s} s)_{V-A}^{j i}\right] k^{\frac{1}{2}} F_{4}\right\}
\end{aligned}
$$

where

$$
\begin{aligned}
& (17): z_{+}=\frac{m_{c}^{2}}{\left(m_{b}+m_{u}\right)^{2}}, k=\frac{\alpha_{s}(\mu)}{\alpha_{s}\left(m_{b}+m_{u}\right)}, \\
& (18): z_{-}=\frac{m_{c}^{2}}{\left(m_{b}-m_{d}\right)^{2}}, k=\frac{\alpha_{s}(\mu)}{\alpha_{s}\left(m_{b}-m_{d}\right)}, \\
& (19): z_{-}=\frac{m_{c}^{2}}{\left(m_{b}-m_{s}\right)^{2}}, k=\frac{\alpha_{s}(\mu)}{\alpha_{s}\left(m_{b}-m_{s}\right)} .
\end{aligned}
$$




\section{Lifetimes of $\Xi_{b c}^{+}, \Xi_{b c}^{0}, \Omega_{b c}^{0}$ baryons}

It can be easily seen that in the case of $\Xi_{b c}^{+}=(b c u)$, $\Xi_{b c}^{0}=(b c d)$, and $\Omega_{b c}^{0}=(b c s)$ baryons both PI and WS channels are opened. As a result, the corresponding transition amplitudes are equal to

$$
\begin{aligned}
& \mathcal{T}_{\Xi_{b c}^{+}}=\mathcal{T}_{35 b}+\mathcal{T}_{35 c}+\mathcal{T}_{\mathrm{PI}}^{\left(\Xi_{b c}^{+}\right)}+\mathcal{T}_{\mathrm{WS}}^{\left(\Xi_{b c}^{+}\right)} \\
& \mathcal{T}_{\Xi_{b c}^{0}}=\mathcal{T}_{35 b}+\mathcal{T}_{35 c}+\mathcal{T}_{\mathrm{PI}}^{\left(\Xi_{b c}^{0}\right)}+\mathcal{T}_{\mathrm{WS}}^{\left(\Xi_{b c}^{0}\right)} \\
& \mathcal{T}_{\Omega_{b c}^{0}}=\mathcal{T}_{35 b}+\mathcal{T}_{35 c}+\mathcal{T}_{\mathrm{PI}}^{\left(\Omega_{b c}^{0}\right)}+\mathcal{T}_{\mathrm{WS}}^{\left(\Omega_{b c}^{0}\right)},
\end{aligned}
$$

where the contributions of the $c$ and $b$ quarks' spectator decays are given in the previous subsections and the PI, WS amplitudes are equal to

$$
\begin{aligned}
& \mathcal{T}_{\mathrm{PI}}^{\left(\Xi_{b c}^{+}\right)}=\mathcal{T}_{\mathrm{PI}, u \bar{d}}^{c}+\mathcal{T}_{\mathrm{PI}, s \bar{c}}^{b}+\mathcal{T}_{\mathrm{PI}, d \bar{u}}^{b}+\sum_{l} \mathcal{T}_{\mathrm{PI}, \bar{\nu} l}^{b}, \\
& \mathcal{T}_{\mathrm{WS}}^{\left(\Xi_{b c}^{+}\right)}=\mathcal{T}_{\mathrm{WS}, b u}+\mathcal{T}_{\mathrm{WS}, b c}, \\
& \mathcal{T}_{\mathrm{PI}}^{\left(\Xi_{b c}^{0}\right)}=\mathcal{T}_{\mathrm{PI}, s \bar{c}}^{b}+\mathcal{T}_{\mathrm{PI}, d \bar{u}}^{b}+\mathcal{T}_{\mathrm{PI}, d \bar{u}}^{b^{\prime}}+\sum_{l} \mathcal{T}_{\mathrm{PI}, l \bar{\nu} l}^{b}, \\
& \mathcal{T}_{\mathrm{WS}}^{\left(\Xi_{b c}^{0}\right)}=\mathcal{T}_{\mathrm{WS}, c d}+\mathcal{T}_{\mathrm{WS}, b c}, \\
& \mathcal{T}_{\mathrm{PI}}^{\left(\Omega_{b c}^{0}\right)}=\mathcal{T}_{\mathrm{PI}, u \bar{d}}^{c^{\prime}}+\sum_{l} \mathcal{T}_{\mathrm{PI}, \nu, \bar{l}}^{c}+\mathcal{T}_{\mathrm{PI}, s \bar{c}}^{b}+\mathcal{T}_{\mathrm{PI}, d \bar{u}}^{b} \\
& +\sum_{l} \mathcal{T}_{\mathrm{PI}, l \bar{l}_{l}}^{b}+\mathcal{T}_{\mathrm{P}, s \bar{c}}^{b^{\prime}}, \\
& \mathcal{T}_{\mathrm{WS}}^{\left(\Omega_{b c}^{0}\right)}=\mathcal{T}_{\mathrm{WS}, b c}+\mathcal{T}_{\mathrm{WS}, c s} .
\end{aligned}
$$

In these expressions [25]

$$
\begin{gathered}
\mathcal{T}_{\mathrm{PI}, s \bar{c}}^{b}=-\frac{G_{F}^{2}\left|V_{c b}\right|^{2}}{4 \pi} m_{b}^{2}\left(1-\frac{m_{c}}{m_{b}}\right)^{2}\left\{\left[G_{1}\left(z_{-}\right)(\bar{b} b)_{V-A}^{i i}(\bar{c} c)_{V-A}^{j j}+G_{2}\left(z_{-}\right)(\bar{b} b)_{A}^{i i}(\bar{c} c)_{V-A}^{j j}\right]\right. \\
\left.\times\left[F_{1}+\frac{1}{3}\left(1-k^{\frac{1}{2}}\right) F_{2}\right]+\left[G_{1}\left(z_{-}\right)(\bar{b} b)_{V-A}^{i j}(\bar{c} c)_{V-A}^{j i}+G_{2}\left(z_{-}\right)(\bar{b} b)_{A}^{i j}(\bar{c} c)_{V-A}^{j i}\right] k^{\frac{1}{2}} F_{2}\right\}, \\
\mathcal{T}_{\mathrm{PI}, d \bar{u}}^{b}=\mathcal{T}_{\mathrm{PI}, s \bar{c}}^{b}\left(z_{-} \rightarrow 0\right), \\
\mathcal{T}_{\mathrm{PI}, \tau \bar{\nu}_{\tau}}^{b}=-\frac{G_{F}^{2}\left|V_{c b}\right|^{2}}{\pi} m_{b}^{2}\left(1-\frac{m_{c}}{m_{b}}\right)^{2}\left[G_{1}\left(z_{\tau}\right)(\bar{b} b)_{V-A}^{i j}(\bar{c} c)_{V-A}^{j i}+G_{2}\left(z_{\tau}\right)(\bar{b} b)_{A}^{i j}(\bar{c} c)_{V-A}^{j i}\right], \\
\mathcal{T}_{\mathrm{WS}, b c}=\frac{G_{F}^{2}\left|V_{c b}\right|^{2}}{4 \pi} m_{b}^{2}\left(1+\frac{m_{c}}{m_{b}}\right)^{2}\left(1-z_{+}\right)^{2}\left[\left(F_{6}+\frac{1}{3}\left(1-k^{\frac{1}{2}}\right) F_{5}\right)(\bar{b} b)_{V-A}^{i i}(\bar{c} c)_{V-A}^{j j}+k^{\frac{1}{2}} F_{5}(\bar{b} b)_{V-A}^{i j}(\bar{c} c)_{V-A}^{j i}\right], \\
\mathcal{T}_{\mathrm{PI}, e \bar{\nu}_{e}}^{b}=\mathcal{T}_{\mathrm{PI}, \mu \bar{\nu}_{\mu}}^{b}=\mathcal{T}_{\mathrm{PI}, \tau \bar{\nu}_{\tau}}^{b}\left(z_{\tau} \rightarrow 0\right),
\end{gathered}
$$

where

$$
\begin{aligned}
& (20): z_{-}=\frac{m_{c}^{2}}{\left(m_{b}-m_{c}\right)^{2}}, k=\frac{\alpha_{s}(\mu)}{\alpha_{s}\left(m_{b}-m_{c}\right)}, \\
& (21): z_{\tau}=\frac{m_{\tau}^{2}}{\left(m_{b}-m_{c}\right)^{2}}, \\
& (22): z_{+}=\frac{m_{c}^{2}}{\left(m_{b}+m_{c}\right)^{2}}, k=\frac{\alpha_{s}(\mu)}{\alpha_{s}\left(m_{b}+m_{c}\right)} .
\end{aligned}
$$

The other functions are defined earlier.

\section{E. Numerical results}

From the results presented above it is clear that in OPE formalism the theoretical predictions of doubly heavy baryons' lifetimes depend on such input parameters as the quark masses, wave function at the origin, etc. In paper [35] the following values of these parameters were used:

$$
V_{c s}=0.9745, \quad V_{c b}=0.04,
$$

$T=0.4 \mathrm{GeV}, \quad\left|\Psi^{d l}(0)\right|^{2}=(2.7 \pm 0.2) \times 10^{-3} \mathrm{GeV}^{3}$,

$m_{s}=0.2 \mathrm{GeV}, \quad m_{c}=1.55 \mathrm{GeV}, \quad m_{b}=5.05 \mathrm{GeV}$.

This choice however leads to the following values of the $\Xi_{c c}^{++}$baryon mass and lifetime:

$$
M_{\Xi_{c c}}=3.478 \mathrm{GeV}, \quad \tau_{\Xi_{c c}^{++}}=0.44 \mathrm{ps} .
$$

These results, unfortunately, disagree with the recent experimental data $[4,36]$

$$
\begin{aligned}
M_{\Xi_{c c}^{++}}^{\exp } & =(3621.40 \pm 0.72 \pm 0.27 \pm 0.14) \mathrm{MeV}, \\
\tau_{\Xi_{c c}^{++}}^{\exp } & =0.256_{-0.022}^{+0.024} \pm 0.014 \mathrm{ps},
\end{aligned}
$$

so some change in parameters is required. It should be noted that the values (24) correspond to constituent quark 

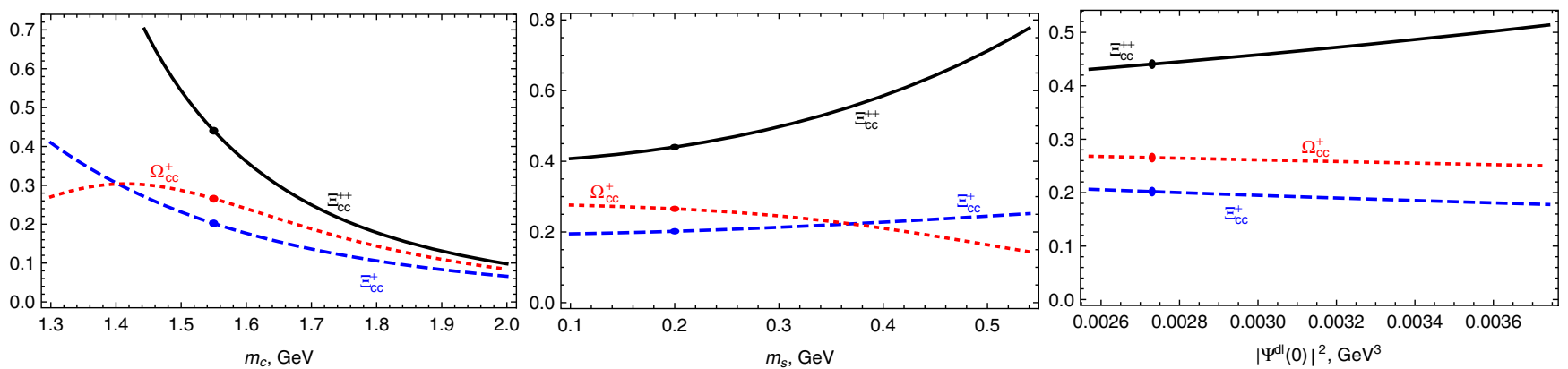

FIG. 5. Lifetimes in ps for $\Xi_{c c}^{++}$(solid black curve), $\Xi_{c c}^{+}$(blue dashed curve) and $\Omega_{c c}^{+}$(red dotted curve) as a function of the model parameters. The results of [35] are shown by dots.
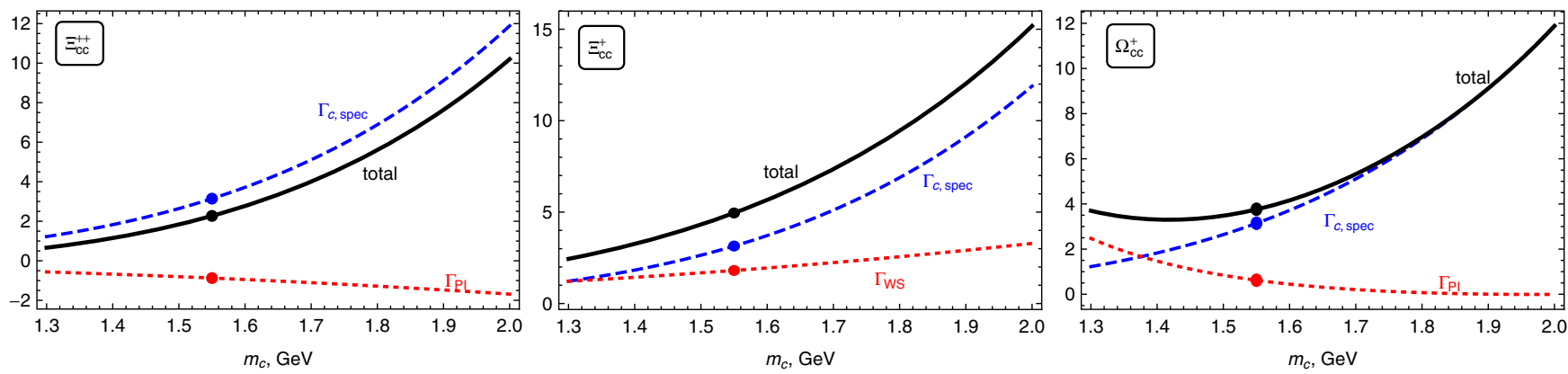

FIG. 6. The partial widths for different operators for doubly charmed baryons (in $\mathrm{ps}^{-1}$ ): operators of dimensions 3 and 5 corresponding to the spectator mechanism (dashed blue curve), operators of dimension 6 corresponding to the weak scattering and Pauli interference (red dotted curve), and the total width (black solid curve). The dots correspond to the predictions of [35].

masses obtained from an analysis of $D$ mesons' lifetimes. In papers $[37,38]$ it was proposed that slightly different masses should be used in the case of doubly heavy baryons. We will discuss the results of these papers in the next subsection, while here we consider quark masses as free and check the dependence of doubly heavy baryon lifetimes on the variation of these parameters.

In Fig. 5 we show the model parameter dependence of the $\Xi_{c c}^{++}$lifetime, while Fig. 6(a) shows the $m_{c}$ dependence of the different channels that contribute to this lifetime. It can be seen from these figures that $\tau\left(\Xi_{c c}^{++}\right)$is most sensitive to a change in the $c$-quark mass. Our analysis shows that the experimental values in (27) are restored with the following values:

$$
m_{c}=1.73 \pm 0.07 \mathrm{GeV}, \quad m_{s}=0.35 \pm 0.2 \mathrm{GeV}
$$

With these masses we have $\tau\left(\Xi_{c c}^{++}\right)=0.26 \pm 0.03$ ps. In the second column of Table I we show calculated with these masses contributions of different decay channels to the $\Xi_{c c}^{++}$ baryon lifetime in comparison with that presented in [35]. One can see from this table that, as mentioned in the previous sections, the spectator decay channel gives the main contribution and it increases with the increase of the charm quark mass. In addition, the PI channel gives a destructive contribution in this case, which leads to an increase of the lifetime. As for the weak scattering mechanism, it is forbidden for $\Xi_{c c}^{++}$decay.

Using the approach described above, it is easy to calculate also the lifetimes of the $\Xi_{c c}^{+}$and $\Omega_{c c}^{+}$baryons:

TABLE I. Lifetimes of doubly charmed baryons and different and partial contributions of different mechanisms (values in brackets correspond to [35]). Theoretical uncertainties are caused by $m_{s, c}$ variation (28).

\begin{tabular}{lccc}
\hline \hline & $\Xi_{c c}^{++}$ & $\Xi_{c c}^{+}$ & $\Omega_{c c}^{+}$ \\
\hline$\sum c \rightarrow s\left(\mathrm{ps}^{-1}\right)$ & $5.1 \pm 0.5(3.1)$ & $5.1 \pm 0.5(3.1)$ & $5.1 \pm 0.5(3.1)$ \\
$\mathrm{PI}\left(\mathrm{ps}^{-1}\right)$ & $-1.2 \pm 0.1(-0.87)$ & 0 & $0.65 \pm 0.5(0.62)$ \\
$\mathrm{WS}\left(\mathrm{ps}^{-1}\right)$ & 0 & $2.3 \pm 0.2(1.8)$ & 0 \\
$\tau(\mathrm{ps})$ & $0.26 \pm 0.03(0.44)$ & $0.14 \pm 0.01(0.2)$ & $0.18 \pm 0.02(0.27)$ \\
\hline \hline
\end{tabular}



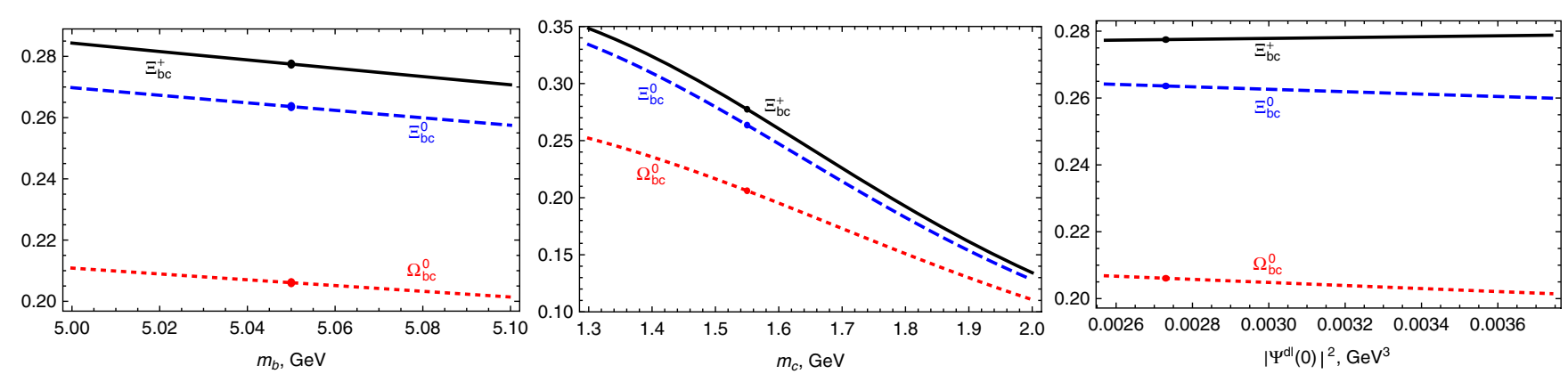

FIG. 7. Lifetimes in ps for $\Xi_{b c}^{+}$(solid black curve), $\Xi_{b c}^{0}$ (blue dashed curve) and $\Omega_{b c}^{0}$ (red dotted curve) as a function of the model parameters. The results of [35] are shown by dots.
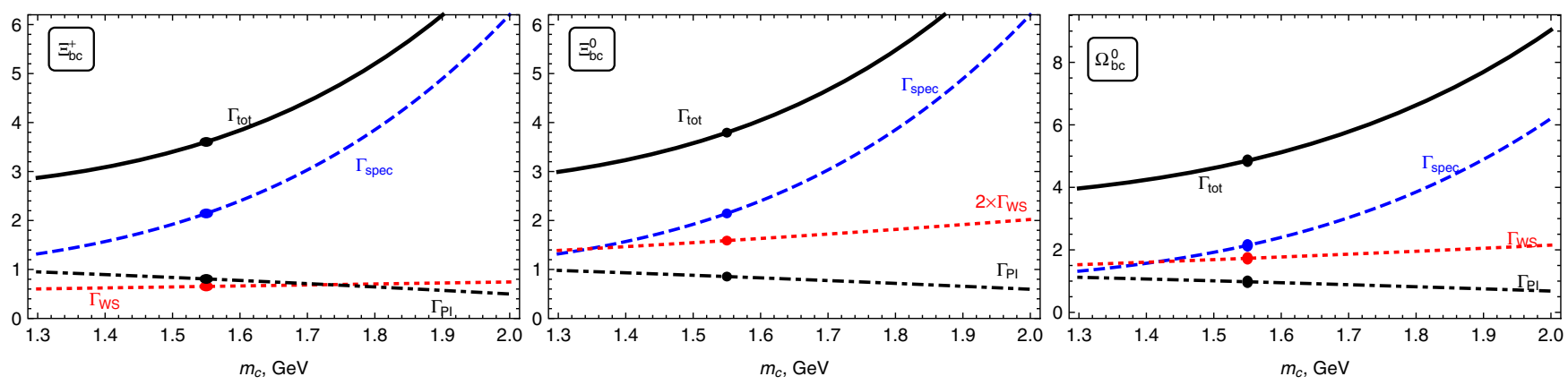

FIG. 8. The partial widths for different operators for $b c$ baryons (in $\mathrm{ps}^{-1}$ ): operators of dimensions 3 and 5 corresponding to the spectator mechanism (dashed blue curve); operators of dimension 6 corresponding to the weak scattering and Pauli interference (red dotted and black dashed-dotted curves, respectively); and the total width (black solid curve). The dots correspond to the predictions of [35].

The lifetime and decay width dependencies on parameters are shown in Figs. 5 and 6. The numerical estimations for parameter values (24) and (28) can be found in the third and fourth columns of Table I. In the case of the $\Xi_{c c}^{+}$baryon the PI channel is forbidden; thus only the spectator decay and the weak scattering give contributions. For for $\Omega_{c c}^{+}$baryon the spectator and PI channels are important. The contribution of the last one is positive. As a result theoretical predictions for the lifetimes of $\Xi_{c c}^{+}$and $\Omega_{c c}^{+}$are smaller than for the $\Xi_{c c}^{++}$particle.

Let us now consider lifetimes of $b c$ baryons $\Xi_{b c}^{+}, \Xi_{b c}^{0}$, and $\Omega_{b c}^{0}$. The lifetime dependencies on parameters are shown in Fig. 7. In the following we will use the constituent value $m_{b}=5.05 \mathrm{GeV}$ for the $b$-quark mass and (28) for $m_{c, s}$. In Fig. 8 we show the $m_{c}$ dependence of different channel contributions for these baryons. The predictions corresponding to parameter values (24) and (28) are given in Table II. From the presented results it is clear that $c$-quark spectator decay is dominant for the considered baryons, while contributions of $b$-quark spectator decay are suppressed by the $V_{c b}$ matrix element. As for dimension 6 operators PI and WS, their contributions are suppressed by a large $b$-quark mass and are small. It is interesting to note, however, that, in contrast to $c c$ baryons, in the case of $b c$ baryons both PI and WS channels are not forbidden for all considered particles.

In the case of $b b$ baryons $\Xi_{b b}^{0}, \Xi_{b b}^{-}$, and $\Omega_{b b}^{-}$spectator $b$-quark decay gives the dominant contribution. As for dimension 6 operators, in complete agreement with OPE selection rules their contributions are suppressed by a large quark mass. As a result, lifetime values presented in Table III are close to each other. It should be noted that,

TABLE II. Decay widths and lifetimes for $b c$ baryons. The meanings of the symbols are the same as in Table I.

\begin{tabular}{lccc}
\hline \hline & $\Xi_{b c}^{+}$ & $\Xi_{b c}^{0}$ & $\Omega_{b c}^{0}$ \\
\hline$\sum b \rightarrow c\left(\mathrm{ps}^{-1}\right)$ & $0.551 \pm 0.0311(0.632)$ & $0.551 \pm 0.0311(0.632)$ & $0.551 \pm 0.0311(0.632)$ \\
$\sum c \rightarrow s\left(\mathrm{ps}^{-1}\right)$ & $2.32 \pm 0.342(1.51)$ & $2.32 \pm 0.342(1.51)$ & $2.32 \pm 0.342(1.51)$ \\
$\mathrm{PI}\left(\mathrm{ps}^{-1}\right)$ & $0.69 \pm 0.044(0.81)$ & $0.75 \pm 0.039(0.86)$ & $0.86 \pm 0.044(0.98)$ \\
WS $\left(\mathrm{ps}^{-1}\right)$ & $0.69 \pm 0.014(0.65)$ & $0.87 \pm 0.022(0.79)$ & $2 . \pm 0.13(1.7)$ \\
$\tau(\mathrm{ps})$ & $0.24 \pm 0.02(0.28)$ & $0.22 \pm 0.018(0.26)$ & $0.18 \pm 0.0088(0.21)$ \\
\hline \hline
\end{tabular}


TABLE III. Decay widths and lifetimes for $b b$ baryons, with designations as in Table I.

\begin{tabular}{lccc}
\hline \hline & $\Xi_{b b}^{0}$ & $\Xi_{b b}^{-}$ & $\Omega_{b b}^{-}$ \\
\hline$\sum b \rightarrow c\left(\mathrm{ps}^{-1}\right)$ & $1.9 \pm 0.0344(1.25)$ & $1.9 \pm 0.0344(1.25)$ & $1.9 \pm 0.0344(1.25)$ \\
$\mathrm{PI}\left(\mathrm{ps}^{-1}\right)$ & 0 & $-0.016 \pm 0.0003(-0.013)$ & $-0.011 \pm 0.0014(-0.01)$ \\
$\mathrm{WS}\left(\mathrm{ps}^{-1}\right)$ & $0.023 \pm 0.00064(0.019)$ & 0 & 0 \\
$\tau\left(\mathrm{ps}^{-1}\right)$ & $0.52 \pm 0.0095(0.79)$ & $0.53 \pm 0.0096(0.81)$ & $0.53 \pm 0.0093(0.8)$ \\
\hline \hline
\end{tabular}

similar to $c c$ sector, different decay mechanisms are enabled for different baryons: WS is enabled only for neutral particles and PI is enabled only for charged ones. Parameter dependence of the lifetimes and decay widths of these baryons is shown in Figs. 9 and 10.

\section{F. Comparison with other works}

One can find in the literature some other theoretical works devoted to the analysis of doubly heavy baryon lifetimes. In the current subsection we will discuss these papers and compare the results presented there with ours.

As mentioned above, in papers [37,38] it was assumed that quark masses used for the analysis of doubly heavy baryons could be a little bit different from the constituent quark masses obtained from the analysis of meson spectroscopy. In particular, in paper [37] ([KR14]) the following values were considered:

$$
\begin{gathered}
m_{q}^{[\mathrm{KR} 14]}=363 \mathrm{MeV}, \quad m_{s}^{[\mathrm{KR} 14]}=538 \mathrm{MeV}, \\
m_{c}^{[\mathrm{KR} 14]}=1.7105 \mathrm{GeV},
\end{gathered}
$$

that correspond to a $\Xi_{c c}^{++}$mass and lifetime equal to

$M_{\Xi_{c c}}^{[\mathrm{KR} 14]}=(3627 \pm 12) \mathrm{MeV}, \quad \tau_{\Xi_{c c}^{++}}^{[\mathrm{KR} 14]}=0.185 \mathrm{ps}$.

One can see that the mass of the baryon is closer to the experimental value (27), while the lifetime is even smaller. We would like, however, to make some comments considering the last result. As presented in [37], the analytical expression for the $\Xi_{c c}^{++}$decay width reads

$$
\Gamma_{\text {tot }}^{[\mathrm{KR} 14]}\left(\Xi_{c c}^{++}\right)=10 \frac{G_{F}^{2} M_{\Xi_{c c}}^{2}}{192 \pi^{3}} f\left(x_{c c}\right), \quad x_{c c}=\frac{M_{\Xi_{c c}}^{2}}{M_{\Xi_{c}}^{2}} .
$$
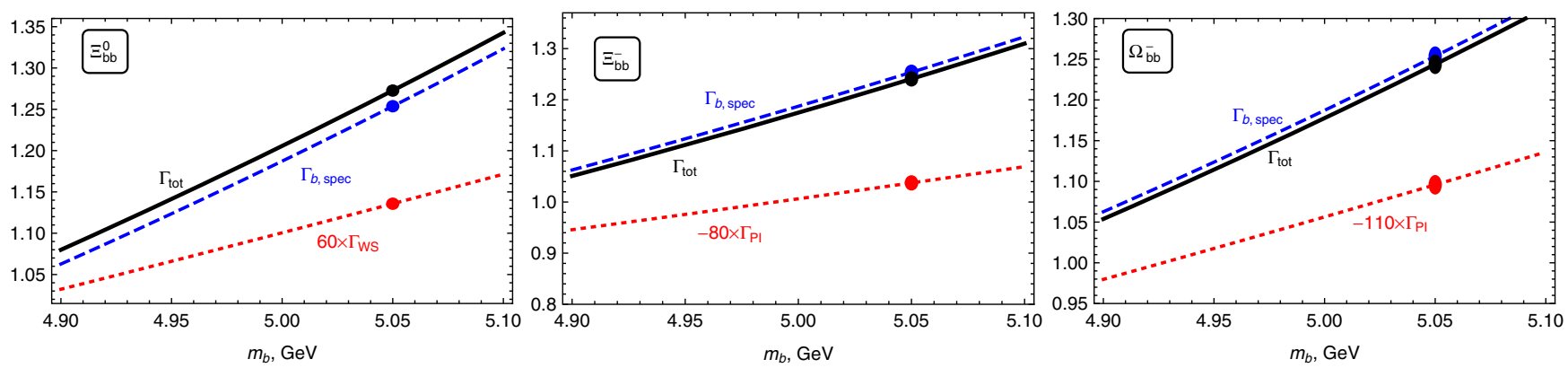

FIG. 9. Decay widths for $b b$ baryons. Designations as in Fig. 6.
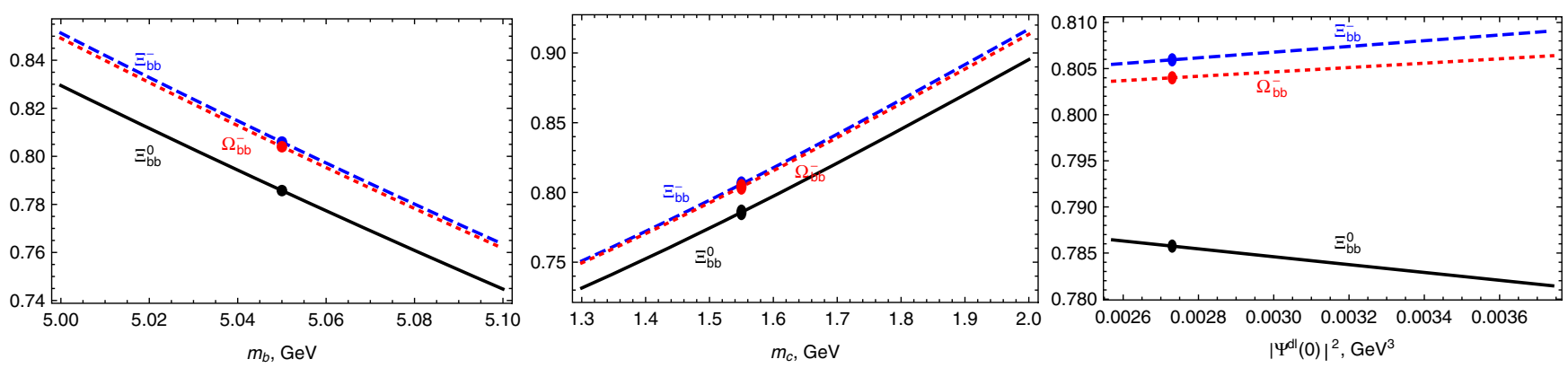

FIG. 10. Lifetimes in ps for $\Xi_{b b}^{0}$ (solid black curve), $\Xi_{b b}^{-}$(blue dashed curve) and $\Omega_{b b}^{-}$(red dotted curve) as a function of the model parameters. The results of [35] are shown by dots. 
From this expression it is clear that in [37] only the spectator decays of the valence $c$ quark contribute. Indeed, the prefactor $10=2 \times(3+1+1)$ in relation (32) shows that only the $c \rightarrow s u d, c \rightarrow s e \nu_{c}$, and $c \rightarrow \mu \nu_{m u}$ channels were taken into account and the final result is doubled because of the two valence quarks in the $\Xi_{c c}$ baryon Fock state. It seems to us that such an approach is not reliable.

First of all, as it can be clearly seen from comparison with the neutron's total width, the factor 2 mentioned above should be avoided. Indeed, since only one spectator decay $d \rightarrow u e \nu_{e}$ is possible in this case and there are two valence $d$ quarks in the neutron, the approach used in [37] would give us the lifetime

$$
\tau_{n}=\left[2 \frac{G_{F}^{2} m_{n}^{5}}{192 \pi^{3}} f\left(\frac{m_{p}^{2}}{m_{n}^{2}}\right)\right]^{-1} \approx 320 \mathrm{~s},
$$

which is almost three times smaller than the experimental result $\tau_{n}^{\exp }=939 \mathrm{~s}$. Without the factor 2 in relation (33) this disagreement is partially removed. In addition, in paper [37] contributions of any form factors are neglected. It is clear that the energy deposit in $\Xi_{c c}$ baryon decay is much larger than for neutron $\beta$-decay. It is well known, however, that even in the latter case $n \rightarrow p e \nu_{e}$ such form factors are important (actually, the axial form factor helps us to obtain the experimental value of the considered lifetime), so it seems strange to forget about them in the case of the $\Xi_{c c}$ lifetime.

The other point is that PI and WS contributions are completely ignored in [37]. As a result, one can expect that the lifetimes of all $c c q, c c s$ baryons should be equal to each other. For some reason, however, the authors of paper [37] use a completely different approach to calculate the $\Xi_{c c}^{+}$ baryon lifetime and the value $\tau_{\Xi_{c c}^{+}} \approx \tau\left(\Xi_{c c}^{++}\right) / 2$ is given there. No detailed explanation for such a difference in calculation methods is presented in [37].

If we use the values presented in [KR14] in the OPE calculations described above, the lifetime of a $\Xi_{c c}^{++}$baryon is equal to $0.32 \mathrm{ps}$, which is a little bit larger than the experimental result (27). In paper [38] ([KR18]) another set of quark masses was presented that describes both meson and baryon masses:

$$
\begin{aligned}
& m_{q}^{[\mathrm{KR} 18]}=308.5 \mathrm{MeV}, \\
& m_{s}^{[\mathrm{KR} 18]}=482.2 \mathrm{MeV}, \\
& m_{c}^{[\mathrm{KR} 18]}=1655.6 \mathrm{GeV} .
\end{aligned}
$$

No predictions for the lifetimes can be found in this paper, but the OPE approach gives the value $\tau\left(\Xi_{c c}^{++}\right) \approx 0.37 \mathrm{ps,}$ which is also larger than the experimental one.

In a series of papers [33,39-41] the lifetimes of heavy and doubly heavy baryons were considered in the framework of operator product expansion with the PI and WS channels taken into account. The results of these works agree qualitatively with ours (for example, the hierarchy of $c c$ baryon lifetimes is the same), but the numerical values of the lifetimes are somewhat larger. The reason for the difference is that the values of the quark masses used in these papers are smaller (for example, $m_{c}=1.35 \mathrm{GeV}$ in these papers).

It should be noted that the mass of a $c$ quark is not really large, so higher order contributions in operator product expansion could also give significant contributions. In the recent article [42] the authors showed that the experimental value of the $\Xi_{c c}^{++}$baryon lifetime can be explained if contributions of higher dimension operators are taken into account. It is interesting to note that the lifetimes of other doubly charmed baryons are changed in different ways in comparison with our results: $\tau\left(\Xi_{c c}^{+}\right)$decreases only slightly, while the lifetime of a $\Omega_{c c}^{+}$baryon increases and is comparable with $\tau\left(\Xi_{c c}^{++}\right)$. It is clear that a detailed theoretical and experimental investigation of the lifetimes of these particles is highly desirable.

\section{OBSERVATION PERSPECTIVES}

Here we briefly discuss the observation possibilities of doubly heavy baryons at the LHC. As already mentioned the LHCb Collaboration observed $\Xi_{c c}^{++}$baryons in the decay mode $\Lambda_{c}^{+} K^{-} \pi^{+} \pi^{+}$[4] and confirmed this observation in the decay mode $\Xi_{c}^{+} \pi^{+}[5]$.

The next step is the observation of $\Xi_{c b}$ baryons. In spite of the large number of theoretical predictions for branching fractions (see, for example, [1,43-48] and Table IV), the

TABLE IV. Branching fractions of the exclusive decays.

\begin{tabular}{lcclclccc}
\hline \hline Mode & {$[1,47]$} & {$[48]$} & \multicolumn{1}{c}{ Mode } & {$[1,47]$} & {$[48]$} & Mode & {$[1,47]$} & {$[48]$} \\
\hline$\Xi_{c c}^{++} \rightarrow \Xi_{c}^{+} \rho^{+}$ & 46.8 & 14.2 & $\Xi_{c c}^{+} \rightarrow \Xi_{c}^{0} \rho^{+}$ & 33.6 & 4.66 & $\Omega_{c c}^{+} \rightarrow \Omega_{c}^{0} \rho^{+}$ & 0 & 24.2 \\
$\Xi_{c c}^{++} \rightarrow \Xi_{c}^{+} \pi$ & 15.7 & 7.24 & $\Xi_{c c}^{+} \rightarrow \Xi_{c}^{0} \pi$ & 11.2 & 2.4 & $\Omega_{c c}^{+} \rightarrow \Omega_{c}^{0} \pi$ & 0 & 7.05 \\
$\Xi_{c c}^{++} \rightarrow \Xi_{c}^{+} \ell \nu_{\ell}$ & 16.8 & 5.39 & $\Xi_{c c}^{+} \rightarrow \Xi_{c}^{0} \ell \nu_{\ell}$ & 7.5 & 1.77 & $\Omega_{c c}^{+} \rightarrow \Omega_{c}^{0} \ell \nu_{\ell}$ & 0 & 6.65 \\
$\Xi_{b c}^{+} \rightarrow \Xi_{b}^{0} \rho^{+}$ & 21.7 & 6.24 & $\Xi_{b c}^{0} \rightarrow \Xi_{b}^{-} \rho^{+}$ & 20.1 & 2.36 & $\Omega_{b c}^{0} \rightarrow \Omega_{b}^{-} \rho^{+}$ & 0 & 18. \\
$\Xi_{b c}^{+} \rightarrow \Xi_{b}^{0} \pi$ & 7.7 & 3.25 & $\Xi_{b c}^{0} \rightarrow \Xi_{b}^{-} \pi$ & 7.1 & 1.23 & $\Omega_{b c}^{0} \rightarrow \Omega_{b}^{-} \pi$ & 0 & 4.57 \\
$\Xi_{b c}^{+} \rightarrow \Xi_{b}^{0} \ell \nu_{\ell}$ & 4.4 & 2.3 & $\Xi_{b c}^{0} \rightarrow \Xi_{b}^{-} \ell \nu_{\ell}$ & 4.1 & 0.867 & $\Omega_{b c}^{0} \rightarrow \Omega_{b}^{-} \ell \nu_{\ell}$ & 0 & 6. \\
$\Xi_{b b}^{0} \rightarrow \Xi_{b c}^{+} \ell \nu_{\ell}$ & 14.9 & 2.59 & $\Xi_{b b}^{b} \rightarrow \Xi_{b c}^{0} \ell \nu_{\ell}$ & 14.9 & 1.68 & $\Omega_{b b}^{b} \rightarrow \Omega_{b c}^{0} \ell \nu_{\ell}$ & 0 & 4.83 \\
$\Xi_{b b}^{0} \rightarrow \Xi_{b c}^{+} \rho^{-}$ & 5.7 & 0.617 & $\Xi_{b b}^{-} \rightarrow \Xi_{b}^{0} \rho^{-}$ & 5.7 & 0.265 & $\Omega_{b b}^{-} \rightarrow \Omega_{b b}^{0} \rho^{-}$ & 0 & 1.25 \\
$\Xi_{b b}^{0} \rightarrow \Xi_{b c}^{+} \pi$ & 2.2 & 0.213 & $\Xi_{b b}^{-} \rightarrow \Xi_{b c}^{0} \pi$ & 2.2 & 0.0854 & $\Omega_{b b}^{-} \rightarrow \Omega_{b c}^{0} \pi$ & 0 & 0.43 \\
\hline \hline
\end{tabular}


"golden mode" has not been found yet. Of course, the greater the branching fraction value, the more chances for the decay mode to be observed. But the decay branchings of intermediate particles are also very important. In addition, as shown in [16], the possibility of the experiment also must be taken into account. For example, each extra track in the final state decreases the registration efficiency. That is why understanding the experiment features is very important when searching for the most promising decay modes. We share the cautious optimism of [16] about the observation of particles in the LHCb data of Run I and Run II, and we also think that in any case $\Xi_{c b}$ will be observed in the LHCb data of Run III.

As for the observation of the $\Xi_{b b}$, we doubt its possibility at the LHC because of the very small production rate.

\section{CONCLUSIONS}

This article is devoted to the theoretical study of the total widths, production rates, and observation probabilities of the doubly heavy baryons.

We briefly discussed the production and the possibility of observation of $\Xi_{b c}$ baryons at the LHC, and we showed that the kinematical features of $\Xi_{b c}$ baryon production and $B_{c}$ meson production are very similar.

The main efforts were made to estimate the lifetimes of doubly heavy baryons in the framework of operator product expansion. We studied the lifetime dependence on the main parameters of this formalism, which are the masses of the $s, c$, and $b$ quarks and the value of the diquark wave function at the origin. We show that the spectator heavy quark decays give the main contribution to the lifetimes of doubly heavy baryons. However, in the case of $\Xi_{c c}$ and $\Omega_{c c}$ baryons the contributions of the higher dimension terms, such as weak scattering and Pauli interference channels, are also important. For $b c q$ and $b b q$ baryons the higher dimension terms are suppressed by the large mass of the heavy quark and do not contribute essentially to the lifetime value.

The lifetime predictions for doubly heavy baryons are most sensitive to the charm quark mass. The knowledge of the experimental value of the $\Xi_{c c}^{++}$baryon lifetime allowed us to determine this parameter with fairly good accuracy and to make the lifetime predictions for other doubly heavy baryons.

\section{ACKNOWLEDGMENTS}

The authors would like to thank V. Galkin, V. V. Kiselev, and A. Onishchenko for help and useful discussions. A. B. acknowledges the support from the Ministry of Education and Science of the Russian Federation of RF (Grant No. 14.610.21.0002, identification number RFMEFI61014X0002); "Basis" Foundation (Grant No. 17-12-244-1); and Russian Foundadtion for Basic Research (Grant No. 19-02-00302).
[1] V. V. Kiselev and A. K. Likhoded, Usp. Fiz. Nauk 172, 497 (2002) [Phys. Usp. 45, 455 (2002)].

[2] C. Albertus, E. Hernandez, J. Nieves, and J. M. Verde-Velasco, Eur. Phys. J. A 31, 691 (2007).

[3] C. Albertus, E. Hernandez, J. Nieves, and J. M. Verde-Velasco, Eur. Phys. J. A 32, 183 (2007); 36, 119(E) (2008).

[4] R. Aaij et al. (LHCb Collaboration), Phys. Rev. Lett. 119, 112001 (2017).

[5] R. Aaij et al. (LHCb Collaboration), Phys. Rev. Lett. 121, 162002 (2018).

[6] A. V. Berezhnoy, V. V. Kiselev, A. K. Likhoded, and A. I. Onishchenko, Phys. Rev. D 57, 4385 (1998).

[7] R. Aaij et al. (LHCb Collaboration), J. High Energy Phys. 06 (2012) 141; 03 (2014) 108(A).

[8] V. V. Kiselev, A. A. Novoselov, and E. R. Tagiev, arXiv: 1612.00444.

[9] S. Dulat, T.-J. Hou, J. Gao, M. Guzzi, J. Huston, P. Nadolsky, J. Pumplin, C. Schmidt, D. Stump, and C. P. Yuan, Phys. Rev. D 93, 033006 (2016).

[10] S. S. Gershtein, V. V. Kiselev, A. K. Likhoded, A. V. Tkabladze, A. V. Berezhnoy, and A. I. Onishchenko, arXiv: hep-ph/9803433.
[11] D. Ebert, R. N. Faustov, V. O. Galkin, and A. P. Martynenko, Phys. Rev. D 66, 014008 (2002).

[12] D. Ebert, R. N. Faustov, and V. O. Galkin, Phys. Rev. D 67, 014027 (2003).

[13] S. Godfrey, Phys. Rev. D 70, 054017 (2004).

[14] D. Ebert, R. N. Faustov, and V. O. Galkin, Eur. Phys. J. C 71, 1825 (2011).

[15] V. O. Galkin (private communication).

[16] S. Blusk, Workshop on heavy hadron spectroscopy, 2017 (CERN, 2017), https://indico.cern.ch/event/646144/timetable/.

[17] M. A. Shifman and M. B. Voloshin, Yad. Fiz. 41, 187 (1985) [Sov. J. Nucl. Phys. 41, 120 (1985)].

[18] M. A. Shifman and M. B. Voloshin, Zh. Eksp. Teor. Fiz. 91, 1180 (1986) [Sov. Phys. JETP 64, 698 (1986)].

[19] M. B. Voloshin, Phys. Lett. B 385, 369 (1996).

[20] B. Guberina, R. Ruckl,, and J. Trampetic, Z. Phys. C 33, 297 (1986).

[21] A. F. Falk, M. E. Luke, M. J. Savage, and M. B. Wise, Phys. Rev. D 49, 555 (1994).

[22] A. V. Berezhnoy, V. V. Kiselev, and A. K. Likhoded, Yad. Fiz. 59, 909 (1996) [Phys. At. Nucl. 59, 870 (1996)].

[23] V. V. Kiselev, A. K. Likhoded, and M. V. Shevlyagin, Phys. Lett. B 332, 411 (1994). 
[24] V. V. Kiselev, A. K. Likhoded, and A. I. Onishchenko, Phys. Rev. D 60, 014007 (1999).

[25] V. V. Kiselev, A. K. Likhoded, and A. I. Onishchenko, Eur. Phys. J. C 16, 461 (2000).

[26] I. I. Y. Bigi, N. G. Uraltsev, and A. I. Vainshtein, Phys. Lett. B 293, 430 (1992); 297, 477(E) (1992).

[27] I. I. Y. Bigi, B. Blok, M. A. Shifman,, and A. I. Vainshtein, Phys. Lett. B 323, 408 (1994).

[28] B. Blok and M. A. Shifman, Nucl. Phys. B399, 441 (1993).

[29] A. V. Manohar and M. B. Wise, Phys. Rev. D 49, 1310 (1994).

[30] A. F. Falk, Z. Ligeti, M. Neubert, and Y. Nir, Phys. Lett. B 326, 145 (1994).

[31] L. Koyrakh, Phys. Rev. D 49, 3379 (1994).

[32] A. I. Onishchenko, arXiv:hep-ph/9912424.

[33] B. Guberina, B. Melic,, and H. Stefancic, Eur. Phys. J. C 9 , 213 (1999); 13, 551 (2000).

[34] B. Guberina, B. Melic,, and H. Stefancic, QCD: Perturbative or nonperturbative?, Proceedings of the 17th Autumn School, Lisbon, Portugal, 1999 (World Scientific, 1999), pp. 363-368.

[35] A. K. Likhoded and A. I. Onishchenko, arXiv:hep-ph/ 9912425.
[36] R. Aaij et al. (LHCb Collaboration), Phys. Rev. Lett. 121, 052002 (2018).

[37] M. Karliner and J. L. Rosner, Phys. Rev. D 90, 094007 (2014).

[38] M. Karliner and J. L. Rosner, Phys. Rev. D 97, 094006 (2018).

[39] B. Guberina and B. Melic, Eur. Phys. J. C 2, 697 (1998).

[40] B. Guberina, B. Melic,, and H. Stefancic, Phys. Lett. B 469, 253 (1999).

[41] B. Guberina, B. Melic,, and H. Stefancic, Phys. Lett. B 484, 43 (2000).

[42] H.-Y. Cheng and Y.-L. Shi, arXiv:1809.08102.

[43] F.-S. Yu, H.-Y. Jiang, R.-H. Li, C.-D. Lü, W. Wang, and Z.-X. Zhao, Chin. Phys. C 42, 051001 (2018).

[44] C. Albertus, E. Hernandez, and J. Nieves, Proc. Sci., QNP2012 (2012) 073.

[45] C. Albertus, E. Hernandez, and J. Nieves, Proc. Sci., QNP2012 (2012) 090.

[46] A. Faessler, T. Gutsche, M. A. Ivanov, J. G. Korner, and V. E. Lyubovitskij, Phys. Lett. B 518, 55 (2001).

[47] A. I. Onishchenko, in Proceedings of the 5th International Workshop on Heavy Quark Physics Dubna, Russia, 2000 (JINR, Dubna, Russia, 2000).

[48] W. Wang, F.-S. Yu, and Z.-X. Zhao, Eur. Phys. J. C 77, 781 (2017). 